\title{
Inclusion of organ deformation in dose calculations
}

\author{
K. K. Brock, ${ }^{\text {a) }}$ D. L. McShan, and R. K. Ten Haken \\ Department of Radiation Oncology, University of Michigan, Ann Arbor, Michigan 48109 \\ S. J. Hollister \\ Department of Biomedical Engineering, University of Michigan, Ann Arbor, Michigan 48109
}

L. A. Dawson and J. M. Balter

Department of Radiation Oncology, University of Michigan, Ann Arbor, Michigan 48109

(Received 8 July 2002; accepted for publication 26 November 2002; published 5 February 2003)

\begin{abstract}
A previously described system for modeling organ deformation using finite element analysis has been extended to permit dose calculation. Using this tool, the calculated dose to the liver during radiotherapy can be compared using a traditional static model (STATIC), a model including rigid body motion (RB), and finally a model that incorporates rigid body motion and deformation (RBD). A model of the liver, consisting of approximately 6000 tetrahedral finite elements distributed throughout the contoured volume, is created from the CT data obtained at exhale. A deformation map is then created to relate the liver in the exhale CT data to the liver in the inhale CT data. Six intermediate phase positions of each element are then calculated from their trajectories. The coordinates of the centroid of each element at each phase are used to determine the dose received. These intermediate dose values are then time weighted according to a population-modeled breathing pattern to determine the total dose to each element during treatment. This method has been tested on four patient datasets. The change in prescribed dose for each patient's actual tumor as well as a simulated tumor of the same size, located in the superior, intermediate, and inferior regions of the liver, was determined using a normal tissue complication model, maintaining a predicted probability of complications of $15 \%$. The average change in prescribed dose from RBD to STATIC for simulated tumors in the superior, intermediate, and inferior regions are 4.0 (range 2.1 to 5.3), -3.6 (range -5.0 to -2.2 ), and -14.5 (range -27.0 to -10.0 ) Gy, respectively. The average change in prescribed dose for the patient's actual tumor was -0.4 Gy (range -4.1 to $1.7 \mathrm{~Gy}$ ). The average change in prescribed dose from RBD to RB for simulated tumors in the superior, intermediate, and inferior regions are -0.04 (range -2.4 to 2.2), 0.2 (range -1.5 to 1.9 ), and 3.9 (range 0.8 to 7.3 ) Gy, respectively. The average change in the prescribed dose for the patient's actual tumor was 0.7 Gy (range 0.2 to $1.1 \mathrm{~Gy}$ ). This patient sampling indicates the potential importance of including deformation in dose calculations. (C) 2003 American Association of Physicists in Medicine.
\end{abstract}

[DOI: $10.1118 / 1.1539039]$

Key words: organ motion, organ deformation, dose calculations, mechanical modeling

\section{INTRODUCTION}

Organ movement and its impact on treatment dose has been the subject of significant research effort over the past few years. While progress has been made in evaluating movement using a rigid body model, the impact of deformation on dose calculation is an area of study that is still in its infancy.

Several studies have investigated the effects of motion on the calculated dose distribution. For example, Killoran et al. used Monte Carlo to simulate the effect of variation in overall tumor position and introduced the concept of Probability of Prescription Dose to determine nonuniform beam margins that lead to a reduction in dose to critical organs. ${ }^{1}$ Mageras et al. used multiple CT scans to estimate the uncertainty in dose delivery and determine confidence limit dose volume histograms (DVH). ${ }^{2}$

Previous studies have shown that motion of the liver associated with breathing typically ranges from $1-3 \mathrm{~cm}^{3}$ Standard treatment planning assumes that the liver is not moving but includes a treatment margin to account for the breathing motion observed under fluoroscopy. Ten Haken et al. demonstrated that by eliminating organ motion due to breathing, the prescribed dose to focal liver cancer, for a specified normal tissue complication probability (NTCP) risk, was increased by 6-8 Gy. ${ }^{4}$ Obtaining a planning CT scan at end exhale and using asymmetric margins to account for breathing motion has also permitted an increased dose to be delivered to focal liver cancer. ${ }^{5}$

Lujan et al. developed a convolution model for evaluating the impact of ventilatory movement on the dose distribution to the liver. ${ }^{6}$ The convolution calculations demonstrated changes in point dose up to $26 \%$ when compared to static dose calculations. This model assumed that the liver moved as a rigid body, however, and motion along other cardinal axes was ignored.

Evidence of liver deformation during breathing has evolved over the past few years. Miller ${ }^{7}$ described the liver using a viscoelastic model, based on original data from Melvin et al. ${ }^{8}$ Yan et al. showed an example of liver defor- 
mation and described a potential infrastructure for incorporating deformation in treatment planning evaluation. ${ }^{9}$ Kruse et al. performed experiments on pig livers, and documented the elasticity. ${ }^{10}$ Balter et al. demonstrated potential compression of the liver during breathing using data from fluoroscopy. ${ }^{3}$ We have recently demonstrated liver deformation between inhale and exhale states using CT models, and described a method for determining the deformation that occurs from the exhale to the inhale state. ${ }^{11}$ The method uses finite element analysis (FEA) to describe the liver volume as tetrahedral elements. Using a linear elastic model, the transformation for each element is determined using boundary conditions applied to the exhale model to create the shape and position of the inhale model.

The purpose of this study is to investigate the importance of including deformation in dose calculations. A comparison of the calculated dose to the liver is made between the standard static planning method (STATIC), the inclusion of rigid body motion only in the inferior-superior (IS) direction (RB), and including motion in all three directions as well as accounting for a change in shape due to the deformation that occurs during breathing (RBD). A simulated tumor is used to determine the dependence of the tumor location on the influence that deformation has in dose calculations.

\section{METHODS AND MATERIALS}

\section{A. Patient data collection}

For this preliminary investigation, data were acquired from four patients. Under a protocol approved by the Internal Review Board at the University of Michigan, computed tomography (CT) scans were acquired at inhale and exhale breath-hold states. The patients' breath was held either voluntarily $(n=1)$ or using active breathing control $(n=3) .{ }^{20}$ The inhale CT scan was obtained at the end of inhale during normal breathing (i.e. not deep inhale). For voluntary breath hold scans, the patient was coached to hold their breath at the end of inhale during normal breathing. For patients' whose breath was held using active breathing control, the breathhold was triggered at end inhale, as determined during normal breathing preceding the breath-hold. All scans were acquired using a helical CT scanner (CT/I, General Electric, Milwaukee WI) using a $3 \mathrm{~mm}$ aperture and a pitch of 2. Both inhale and exhale CT scans were obtained in the same way to eliminate any offset between the two datasets due to patient movement (the total time for both scans $\sim 3-5 \mathrm{~min}$ ). Movement of the diaphragm during normal breathing (observed from CT data) ranged from 1.5-2.4 cm. Similar SI movement was seen on fluoroscopy during free breathing.

\section{B. Dose matrix creation}

The attending physician contoured the liver tumor and a plan was created for radiation treatment based on the exhale CT-based patient model, using an in-house three-dimensional treatment planning system (UMPLAN). Planning target volume (PTV) margins included an isotropic $5 \mathrm{~mm}$ expansion for setup error, an inferior margin for the patient-specific (a)

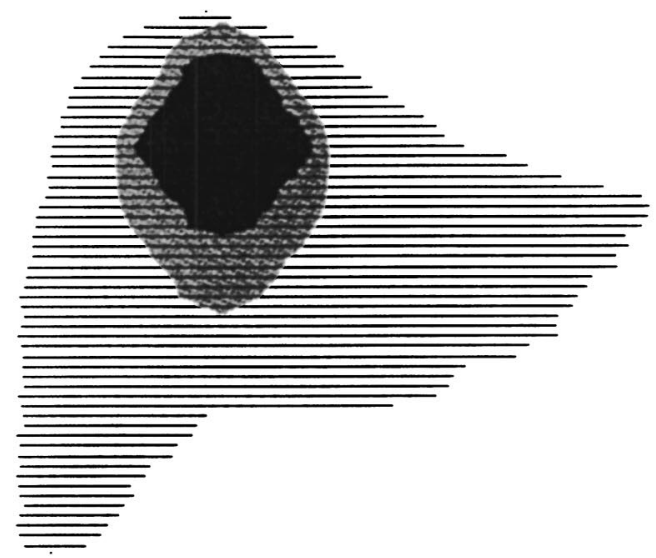

(b)

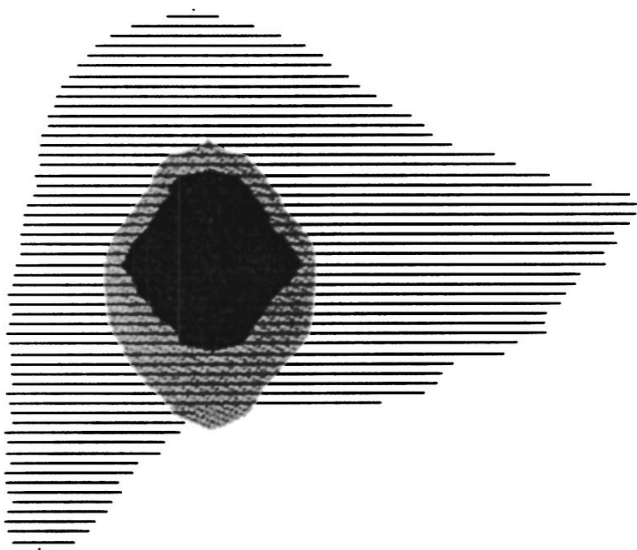

(c)

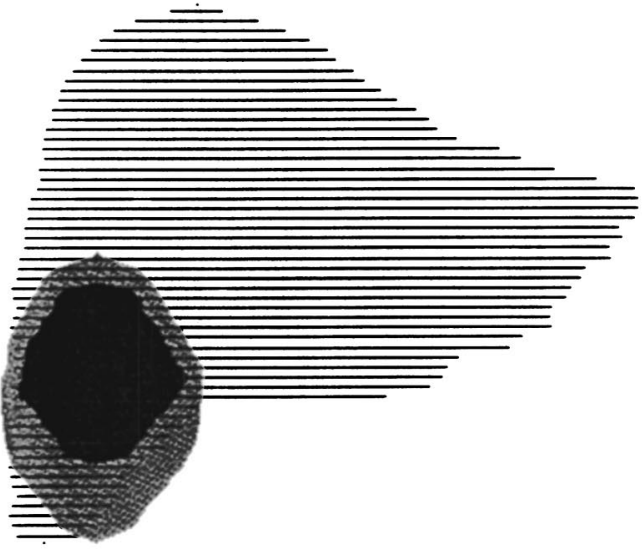

FIG. 1. Simulated tumor (black) and PTV (gray) in the superior (a), intermediate (b), and inferior (c) regions of the liver (lines).

range of diaphragm movement due to breathing, and an additional $3 \mathrm{~mm}$ superior expansion for reproducibility of the exhale state. ${ }^{5}$ For this study, three simulated tumors were also created in the exhale patient dataset. The size of the simulated tumors varied slightly between individual patients to accommodate complete inclusion within the inferior region of the liver. Simulated tumors ranged in volume from 59 to $75 \mathrm{~cm}^{3}$. The simulated tumors were placed in the superior, intermediate, and inferior regions of the liver. Simulated tumors are shown for one case in Fig. 1. Tumors in the 


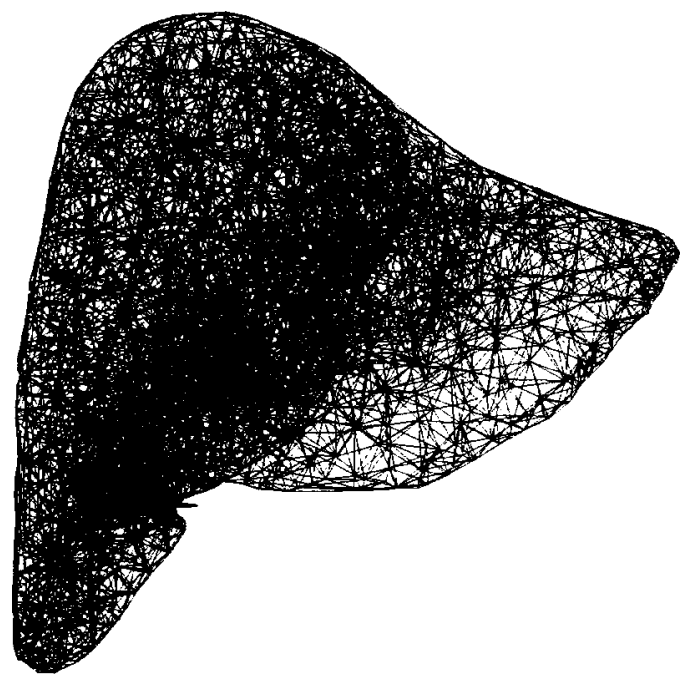

FIG. 2. Tetrahedral mesh of the liver volume.

intermediate region were placed midway within the inferiorsuperior extent of the liver so that the GTV was completely encompassed within the boundaries of the liver, although the PTV expansions were permitted to be outside the liver. The simulated tumors allowed an investigation into the dependence of deformation-induced dosimetric changes on the location of the tumor within the liver.

Treatment plans were created for each of the three simulated tumors, adhering to the same criteria as the original plan for the actual tumor (coverage of the PTV with $95 \%$ or greater dose, constraints on spinal cord dose, and attempts to minimize dose-volume involvement of a normal liver). Dose distributions were calculated on the exhale and inhale CT datasets. The dose matrix had an isotropic spatial resolution of $3 \mathrm{~mm}$.

\section{Dose summation method}

As described previously ${ }^{11}$ the liver model is comprised of 6000 tetrahedral elements of varying size and shape. An example of one liver comprised of such elements is shown in Fig. 2. The volume of each element was computed as well as the location of the centroid. A deformation map was created using either manual or more automated techniques. ${ }^{12}$ Using this deformation map, the displacement of each node that makes up each element in the finite element model was determined. This displacement was then split into six uniform increments to simulate phases of the breathing cycle. Phase 0 corresponded to the exhale position and phase 1 corresponded to the inhale position. The size and centroid location of each element was then determined for each intermediate phase.

The inhale and exhale CT models provided two maps of density at the extremes of normal breathing from which the dose was calculated. While an ideal system for calculating the dose to the intermediate phases of breathing would generate density maps for these states, an approximation was
TABLE I. Relative time weights.

\begin{tabular}{lc}
\hline \multicolumn{1}{c}{$\phi$} & Time weight \\
\hline 0 (exhale) & 0.48 \\
0.2 & 0.13 \\
0.4 & 0.09 \\
0.6 & 0.08 \\
0.8 & 0.10 \\
1.0 (inhale) & 0.12 \\
\hline
\end{tabular}

developed to use the existing data and to modulate the weight of dose calculations from the exhale toward the inhale model as breathing progresses.

The location of the centroid of each element was used to look up the dose at that point in the dose matrix. The relative dose value for a given element and breathing phase was calculated as

$$
D_{\phi}(x, y, z)=D^{E}(x, y, z) \phi+D^{I}(x, y, z)(1-\phi),
$$

where $x, y, z$ are the element coordinates, $D_{\phi}$ is the relative dose at phase $\phi$ (without considerations of time weighting), and $D^{E}$ and $D^{I}$ are the calculated dose values from the exhale and inhale CT density grids, respectively. $\phi$ varies from 0 (exhale) to 1 (inhale) in increments of 0.2 .

Time weighting was then applied to the relative dose at each phase for each element location using information gathered via fluoroscopy on a population of patients previously studied. ${ }^{5}$ The time weighting factors are shown in Table I.

An average volume for each element was also created by time weighting the volume of each element according to the same method described in Table I. This process provided appropriate volume weighting for each element in the construction of dose volume histograms.

\section{Planning methods}

Three calculation methods were compared. The first was the standard static plan (STATIC). The static plan only included the data from phase 0 , the exhale position.

The second method included rigid body (RB) motion in the inferior superior direction only. The motion in the inferior superior (IS) direction was determined from the difference in the position of the diaphragm on the CT scan. This displacement was then assigned as the displacement for every node in the liver in the IS direction. The lateral and AP displacements were set to zero.

The third method included rigid body plus deformation (RBD). This method took advantage of all data obtained from deformation modeling, including varying displacements in all three directions for all elements in the model.

\section{E. Comparison method}

The three planning methods were then compared via observation of dose volume histograms (DVHs) of the normal liver tissue [liver minus gross tumor volume (GTV)], as well as evaluations of effective volume $\left(V_{\text {eff }}\right)^{13}$ of the normal liver volume and change in prescribed dose for a specified normal tissue complication probability (NTCP) risk, ${ }^{14}$ calculated us- 

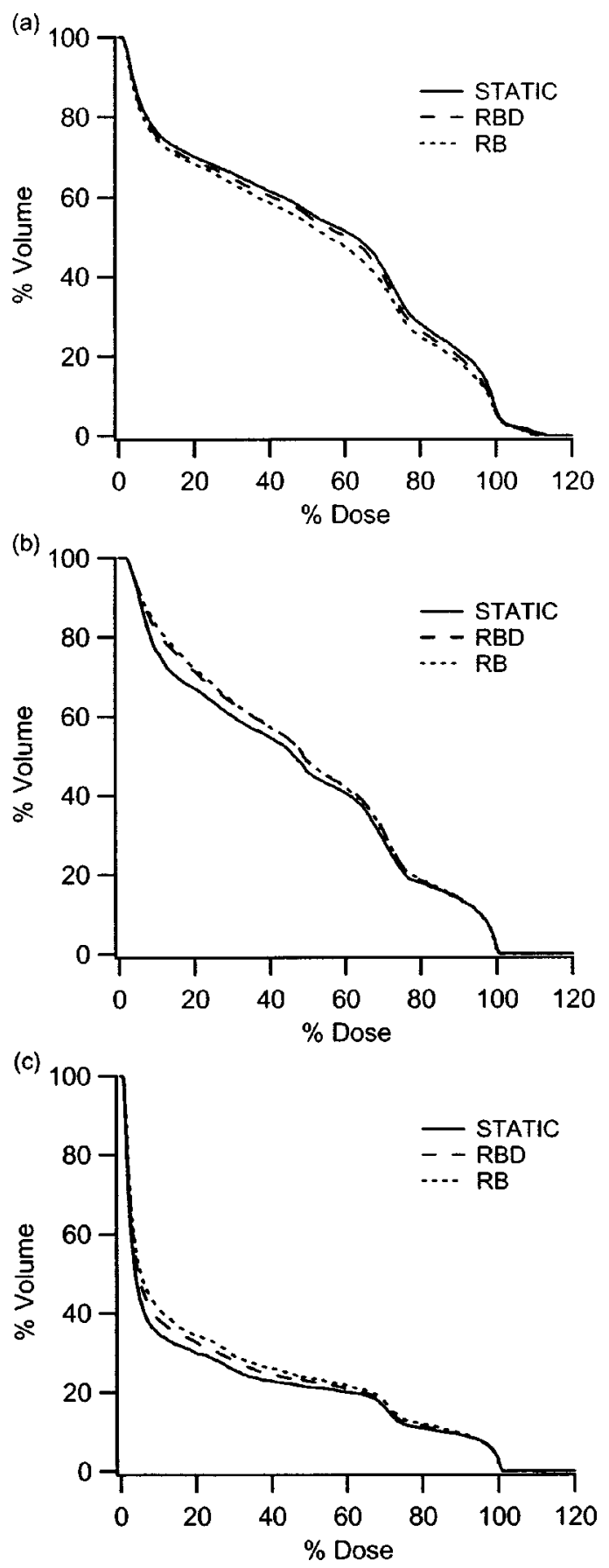

FIG. 3. DVHs for a simulated tumor in the superior (a), intermediate (b), and inferior (c) region of the liver.

ing the Lyman model ${ }^{15}$ with parameters $m=0.12, \mathrm{TD}(1)=46$ Gy, and $n=0.97 .{ }^{16}$ While the current focal liver protocol at the University of Michigan limits the prescribed dose to the tumor to $90 \mathrm{~Gy}$, this limitation was ignored for the simulated tumors to permit comparisons in the event that higher doses were permissible at a 15\% NTCP risk level. Maximum point dose differences between the three methods were also determined. The planning methods were compared for each of the three simulated tumor positions as well as for the actual patient tumor.
TABLE II. Change in prescribed dose $(\mathrm{Gy})$ for $\mathrm{NTCP}=15 \%$ for simulated tumors.

\begin{tabular}{lcccccccc}
\hline \hline & \multicolumn{2}{c}{ Superior } & & \multicolumn{2}{c}{ Intermediate } & & \multicolumn{2}{c}{ Inferior } \\
\cline { 2 - 3 } \cline { 7 - 8 } \cline { 7 - 8 } \cline { 7 - 8 } & AVG & Range & & AVG & Range & & AVG & Range \\
\hline RBD-STATIC & 4.0 & $2.1-5.3$ & & -3.6 & $-5.0--2.2$ & & -14.5 & $-27--10$ \\
RBD-RB & -0.04 & $-2.4-2.2$ & & 0.2 & $-1.5-1.9$ & & 3.9 & $0.8-7.3$ \\
\hline \hline
\end{tabular}

\section{RESULTS}

\section{A. Dose differences}

Differences in calculated dose points were observed between all three methods of dose calculation, STATIC, RB, and RBD. Point dose differences up to $44 \%$ were seen between the STATIC and RBD plans and up to $42 \%$ between the RBD and RB plans. The largest point dose differences were seen along beam edges, in areas of high dose gradients. STATIC, RB, and RBD cumulative DVHs of the normal liver tissue are shown for simulated tumors in the superior, intermediate, and inferior regions of the liver in Fig. 3, respectively, for one example case.

\section{B. Change in prescribed dose-Simulated tumors}

Changes in the tumor prescribed dose, with a liver isoNTCP of $15 \%$, are shown in Table II for the simulated tumors. The average change in prescribed dose between the RBD and STATIC calculations for tumors in the superior, intermediate, and inferior regions of the liver are 4.0 (range 2.1 to 5.3 ), -3.6 (range -5.0 to -2.2 ), and -14.5 (range -27.0 to -10.0$) \mathrm{Gy}$, respectively. The average change in prescribed dose between the RBD and RB calculations for tumors in the superior, intermediate, and inferior regions of the liver are -0.04 (range -2.4 to 2.2 ), 0.2 (range -1.5 to 1.9), and 3.9 (range 0.8 to 7.3) Gy, respectively. The average dose that could be prescribed with an iso-NTCP of $15 \%$, for all patients and all three calculation methods, to the simulated tumors in the superior, intermediate, and inferior regions of the liver, was 84.6, 92.7, and 157.0 Gy.

Changes in the $V_{\text {eff }}$ of normal liver tissue are shown in Table III for the simulated tumors. The average change in $V_{\text {eff }}$ between the RBD and STATIC calculations for tumors in the superior, intermediate, and inferior regions of the liver are $-2.3 \%$ (range -3.1 to $-1.4 \%$ ), $1.7 \%$ (range 1.0 to $2.2 \%$ ), and $2.1 \%$ (range 1.7 to $2.5 \%$ ), respectively. The average change in $V_{\text {eff }}$ between the RBD and RB calculations for tumors in the superior, intermediate, and inferior regions of the liver are $0.1 \%$ (range -1.2 to $1.5 \%$ ), $-0.1 \%$ (range -0.9 to $0.6 \%$ ), and $-0.6 \%$ (range -1.0 to $-0.2 \%$ ), respectively.

\section{Change in prescribed dose-Actual patient tumors}

Changes in the tumor prescribed dose, with a liver isoNTCP of 15\%, are shown in Table IV for the actual patient tumors. The average change in prescribed dose between the RBD and STATIC calculations for the actual tumors is -0.4 (range -4.1 to 1.7 ) Gy. The average change in prescribed 
TABLE III. Change in effective volume $(\%)$ for simulated tumors.

\begin{tabular}{|c|c|c|c|c|c|c|}
\hline & \multicolumn{2}{|c|}{ Superior } & \multicolumn{2}{|c|}{ Intermediate } & \multicolumn{2}{|c|}{ Inferior } \\
\hline & AVG & Range & AVG & Range & AVG & Range \\
\hline RBD-STATIC & -2.3 & $-3.1-1.4$ & 1.7 & $1.0-2.2$ & 2.1 & $1.7-2.5$ \\
\hline RBD-RB & 0.1 & $-1.2-1.5$ & -0.1 & $-0.9-0.6$ & -0.6 & $-1.0--0.2$ \\
\hline
\end{tabular}

dose between the RBD and RB calculations for the actual tumors is 0.7 (range 0.2 to 1.1 ) Gy. The average prescribed dose to the actual tumors was $62.3 \mathrm{~Gy}$.

Changes in the $V_{\text {eff }}$ of normal liver tissue are shown in Table $\mathrm{V}$ for the actual patient tumors. The average change in $V_{\text {eff }}$ between the RBD and STATIC calculations for the actual tumor is $0.2 \%$ (range -2.0 to $3.3 \%$ ). The average change in $V_{\text {eff }}$ between the RBD and RB calculations for the actual tumors is $-0.7 \%$ (range -0.9 to $-0.4 \%$ ).

\section{DISCUSSION}

We have demonstrated an infrastructure to calculate the dose to a deforming organ. Preliminary results show that the inclusion of deformation can make a substantial impact in the prescribed dose to liver tumors, compared to a static dose calculation and calculations including rigid body motion in the inferior-superior direction only. A dose difference dependence on tumor location was observed. Compared to the RBD calculation, the STATIC calculation tended to underprescribe to tumors in the superior region of the liver and overprescribe to tumors in the intermediate and inferior regions of the liver, as expected for those patients planned at exhale. ${ }^{6}$ The tumors in the intermediate region of the liver behaved more like the tumors in the inferior region, because some of the PTV extended outside (below) the liver volume, in a similar manner to tumors in the inferior region of the liver.

Compared to RBD calculations, the RB calculations tended to underprescribe to tumors in the inferior region of the liver. Trends were not seen, between the RBD and RB calculations, for tumors in the superior and intermediate regions of the liver. However, clinically significant differences (defined as a change in the prescribed dose of one fraction, $1.5 \mathrm{~Gy}$, or greater) were seen in two out of four cases for simulated tumors in the superior and intermediate regions of the liver.

The volume of normal tissue treated to a high dose for simulated tumors in the inferior region of the liver is very small, resulting in relatively large changes in prescribed doses (the last column of Table II). This is a consequence of the small effective volume irradiated and the fact that a small

TABLE IV. Change in prescribed dose $(\mathrm{Gy})$ for $\mathrm{NTCP}=15 \%$ for actual tumor.

\begin{tabular}{lcc}
\hline \hline & AVG & \multicolumn{1}{c}{ Range } \\
\hline RBD-STATIC & -0.4 & $-4.1-1.7$ \\
RBD-RB & 0.7 & $0.2-1.1$ \\
\hline \hline
\end{tabular}

TABLE V. Change in effective volume (\%) for actual tumor.

\begin{tabular}{lrl}
\hline \hline & AVG & \multicolumn{1}{c}{ Range } \\
\hline RBD-STATIC & 0.2 & $-2.0-3.3$ \\
RBD-RB & -0.7 & $-0.9--0.4$ \\
\hline \hline
\end{tabular}

change in $V_{\text {eff }}$ can lead to large changes in the normalization dose in this iso-NTCP region. ${ }^{14}$ These high doses were only used to exemplify the simulated difference in the prescribed dose for tumors in the inferior region of the liver. Current focal liver protocols at the University of Michigan have a dose limit of $90 \mathrm{~Gy}$.

One limitation of this method is the lack of intermediate density grids for dose calculations. Ideally six CT scans would be obtained at the six phases of the breathing cycle, where the dose is calculated. However, the accuracy in obtaining a CT scan at a precise point of mid-ventilation is uncertain. Use of active breathing control devices offers some potential, although accuracy at mid-ventilation positions has not yet been investigated.

These findings demonstrate the potential for deformation to impact the calculation of dose to intrahepatic targets, potentially to the extent that clinical decisions about the treatment regime and dose would be impacted. A larger scale study is warranted in order to determine whether any trends can be established as guidelines for the necessity of including deformation in treatment planning for the liver. A more complete understanding of physiologic processes such as breathing and their impact on delivered doses will aid clinics in making decisions related to not only treatment dose prescriptions, but also the relative gains of interventional technology such as gating, ${ }^{17,18}$ active breathing control, ${ }^{19,20}$ and image guidance..$^{21,22}$

\section{ACKNOWLEDGMENTS}

This work was supported in part by NIH Grant No. P01CA59827. The authors would like to thank Robin Marsh for her assistance with treatment planning and Marc Kessler for his valuable advice. The authors would also like to thank Mihaela Rosu for her assistance with NTCP calculations.

${ }^{a)}$ Electronic mail: kkbrock@umich.edu

${ }^{1}$ J. H. Killoran, H. K. Kooy, D. J. Gladstone, F. J. Welte, and C. J. Beard, "A numerical simulation of organ motion and daily setup uncertainties: implications for radiation therapy," Int. J. Radiat. Oncol., Biol., Phys. 37, 213-221 (1997).

${ }^{2}$ G. S. Mageras, G. H. Kutcher, S. A. Leibel, M. J. Zelefsky, E. Melian, R. Mohan, and Z. Fuks, "A method of incorporating organ motion uncertainties into three-dimensional conformal treatment plans," Int. J. Radiat. Oncol., Biol., Phys. 35, 333-342 (1996).

${ }^{3}$ J. M. Balter, L. A. Dawson, S. Kazanjian, C. McGinn, K. K. Brock, T. S. Lawrence, and R. K. Ten Haken, "Determination of ventilatory liver movement via radiographic evaluation of diaphragm position," Int. J. Radiat. Oncol., Biol., Phys. 51, 267-270 (2001).

${ }^{4}$ R. K. Ten Haken, J. M. Balter, L. H. Marsh, J. M. Robertson, and T. S. Lawrence, "Potential benefits of eliminating planning target volume expansions for patient breathing in the treatment of liver tumors," Int. J. Radiat. Oncol., Biol., Phys. 38, 613-617 (1997).

${ }^{5}$ J. M. Balter, K. L. Lam, C. J. McGinn, T. S. Lawrence, and R. K. Ten Haken, "Improvement of CT-based treatment-planning models of ab- 
dominal targets using static exhale imaging," Int. J. Radiat. Oncol., Biol., Phys. 41, 939-943 (1998).

${ }^{6}$ A. E. Lujan, E. W. Larsen, J. M. Balter, and R. K. Ten Haken, "A method for incorporating organ motion due to breathing into 3D dose calculations," Med. Phys. 26, 715-720 (1999).

${ }^{7}$ K. Miller, "Constitutive modeling of abdominal organs," J. Biomech. 33, 367-373 (2000).

${ }^{8}$ J. W. Melvin, R. L. Stalnaker, and V. L. Roberts, "Impact injury mechanisms in abdominal organs," SAE Trans. 730968, 115-126 (1973).

${ }^{9}$ D. Yan and D. Lockman, "Organ/patient geometric variation in external beam radiotherapy and its effects," Med. Phys. 28, 593-602 (2001).

${ }^{10}$ S. A. Kruse, J. A. Smith, A. J. Lawrence, M. A. Dresner, A. Manduca, J. F. Greenleaf, and R. L. Ehman, "Tissue characterization using magnetic resonance elastography: preliminary results," Phys. Med. Biol. 45, 15791590 (2000).

${ }^{11}$ K. K. Brock, S. J. Hollister, L. A. Dawson, and J. M. Balter, "Technical Note: Creating a 4-Dimensional model of the liver using finite element analysis," Med. Phys. 29, 1403-1405 (2002).

${ }^{12}$ C. R. Meyer, J. L. Boes, B. Kim, P. H. Bland, K. R. Zasadny, P. V. Kison, K. Koral, K. A. Frey, and R. L. Wahl, "Demonstration of accuracy and clinical versatility of mutual information for automatic multimodality image fusion using affine and thin-plate spline warped geometric deformations," Med. Image Anal 3, 195-206 (1997).

${ }^{13}$ G. J. Kutcher and C. Burman, "Calculation of complication probability factor for nonuniform normal tissue irradiation: The effective volume method,” Int. J. Radiat. Oncol., Biol., Phys. 16, 1623-1630 (1989).

${ }^{14}$ R. K. Ten Haken, M. K. Martel, M. L. Kessler, M. B. Hazuka, T. S. Lawrence, J. M. Robertson, A. T. Turrisi, and A. S. Lichter, "Use of Veff and iso-NTCP in the implementation of dose escalation protocols," Int. J. Radiat. Oncol., Biol., Phys. 27, 689-695 (1993).
${ }^{15}$ J. T. Lyman, "Complication probability as assessed from dose-volume histograms," Radiat. Res. 104, s13-s19 (1985).

${ }^{16}$ L. A. Dawson, D. Normelle, J. M. Balter, C. J. McGinn, T. S. Lawrence, and R. K. Ten Haken, "Analysis of radiation induced liver disease using the Lyman NTCP model," Int. J. Radiat. Oncol., Biol., Phys. 53, 810821 (2002).

${ }^{17}$ H. D. Kubo and B. C. Hill, "Respiration gated radiotherapy treatment: A technical study," Phys. Med. Biol. 41, 83-91 (1996).

${ }^{18}$ E. C. Ford, G. S. Mageras, E. Yorke, K. E. Rosenzweig, R. Wagman, and C. C. Ling, "Evaluation of respiratory movement during gated radiotherapy using film and electronic portal imaging," Int. J. Radiat. Oncol., Biol., Phys. 52, 522-531 (2002).

${ }^{19}$ J. W. Wong, M. B. Sharpe, D. A. Jaffray, V. R. Kini, J. M. Robertson, J. S. Stromberg, and A. A. Martinez, "The use of active breathing control $(\mathrm{ABC})$ to reduce margin for breathing motion," Int. J. Radiat. Oncol., Biol., Phys. 44, 911-919 (1999).

${ }^{20}$ L. A. Dawson, K. K. Brock, S. Kazanjian, D. Fitch, C. J. McGinn, T. S. Lawrence, R. K. Ten Haken, and J. Balter, "The reproducibility of organ position using active breathing control (ABC) during liver radiotherapy," Int. J. Radiat. Oncol., Biol., Phys. 51, 1410-1421 (2001).

${ }^{21}$ H. Shirato, S. Shimizu, K. Kitamura, T. Nishioka, K. Kagei, S. Hashimoto, H. Aoyama, T. Kunieda, N. Shinohara, H. Dosaka-Akita, and K. Miyasaka, "Four-dimensional treatment planning and fluoroscopic realtime tumor tracking radiotherapy for moving tumor," Int. J. Radiat. Oncol., Biol., Phys. 48, 435-442 (2000).

${ }^{22} \mathrm{~K}$. Kitamura et al., "Registration accuracy and possible migration of internal fiducial gold marker implanted in prostate and liver treated with real-time tumor-tracking radiation therapy (RTRT)," Radiother. Oncol. 62, 275-281 (2002). 